\title{
LETTERS
}

\section{Health Literacy During Aging}

\author{
Madeleine I. Matthiesen, $\mathrm{MD}^{7}$, Monica B. Vela, $\mathrm{MD}^{2}$, and Valerie G. Press, $M D, M P H^{2}$ \\ 'Medicine-Pediatrics, Massachusetts General Hospital, Boston, MA, USA; ${ }^{2}$ Department of Medicine, University of Chicago, Chicago, IL, USA.
}

$\mathrm{J}$ Gen Intern Med 30(12): 1731

DOI: $10.1007 / \mathrm{s} 11606-015-3487-\mathrm{z}$

(c) Society of General Internal Medicine 2015

$\mathrm{T}$ o the Editors:

We applaud Kobayashi et al.'s recent publication entitled, "Cognitive Function and Health Literacy Decline in a Cohort of Aging English Adults," addressing the relationship between age and health literacy, inclusive of cognitive functioning. Given the high prevalence of poor health literacy among older adults, ${ }^{1}$ identifying risk factors and potential opportunities for intervention is critical to improving health care in this vulnerable population.

We note, however, that poor vision is an additional risk factor that may be critical to developing effective interventions for older patients. While Kobayashi et al. acknowledged "sight difficulties" as a reason for non-completion of the health literacy assessment, excluding participants based on sight may have important implications for future study and patient impact. The patient's degree of vision loss was not documented and neither the vision assessment tool nor the parameters of vision loss were identified. Further, the results of participants with vision loss themselves differed across waves, from $1.5 \%$ $(132 / 8780)$ in wave 2 to $1.8 \%(96 / 5330)$ in wave 5. Although the percentages within each group were fairly similar, it is not clear whether these represent the same or different participants, or if the criteria changed over the course of the study.

Regardless of the tool used or changes across time, the percentage of poor vision identified in this population may significantly underestimate the actual proportion of older patients with poor vision. In a national survey of American adults aged 65 years and older, $21 \%$ self-identified as having vision impairment. ${ }^{2}$ Further, at our institution, we have found that nearly half of hospitalized participants 65 years and older have poor vision (compared to about one-quarter among participants under 65$)^{3,4}$

This is salient, because both the health literacy assessment and the cognitive battery require participants to be able to see text. In prior research, we documented that patients 65 years and older have a dual risk for poor health literacy and poor vision when compared to their younger counterparts. ${ }^{4}$ Because older adults with poor vision have a higher risk of falls, delirium, and hastened physical and psychological decline, ${ }^{5}$ it may be important to determine whether vision mitigates some of the demonstrated association between age, health literacy, and cognitive changes. Therefore, future work should avoid the exclusion of aging adults with poor vision and should attempt to further clarify the interaction of these risk factors in order to tailor appropriate interventions.

Corresponding Author: Valerie G. Press, MD,MPH; Department of Medicine, University of Chicago, 5841 S. Maryland Avenue, MC 5000, Chicago, IL 60637, USA (e-mail: vpress@bsd.uchicago.edu).

\section{REFERENCES}

1. Kobayashi LC, Wardle J, Wolf MS, von Wagner C. Cognitive Function and Health Literacy Decline in a Cohort of Aging English Adults. J Gen Int Med. 2015;958-64.

2. Horowitz A, Brennan M, Reinhardt JP. Prevalence and risk factors for selfreported visual impairment among middle-age and older adults. Res Aging. 2005;27(3):307-26.

3. Press VG, Matthiesen MI, Ranadive A, Hariprasad SM, Meltzer DO, Arora VM. Insights into inpatients with poor vision: A high value proposition. J Hosp Med. 2015;10(5):311-3.

4. Press VG, Shapiro MI, Mayo AM, Meltzer Do, Arora VM. More than meets the eye: relationship between low health literacy and poor vision in hospitalized patients. J Health Commun. 2013;18(Suppl 1):197-204.

5. Keller BK, Morton JL, Thomas VS, Potter JF. The effect of visual and hearing impairments on functional status. J Am Geriatr Soc. 1999;47(11): 1319-25. 